\title{
Predicting Readmission Risk Following Percutaneous Coronary Intervention at the Time of Admission
}

\author{
Zaher Fanari, M.D ${ }^{1,2}$, Daniel Elliott, M.D., M.S.C.E. ${ }^{3,4}$, Carla A. Russo ${ }^{4}$, Paul Kolm, Ph.D. ${ }^{4}$, \\ and William S. Weintraub, M.D. ${ }^{1,4}$ \\ ${ }^{1}$ Section of Cardiology, Christiana Care Health System, Newark, DE \\ 2Division of Cardiology, University of Kansas School of Medicine \\ ${ }^{3}$ Department of Medicine, Christiana Care Health System, Newark, DE \\ ${ }^{4}$ Value Institute, Christiana Care Health System, Newark, DE
}

\section{Abstract}

Objective-To investigate whether a prediction model based on data available early in Percutaneous Coronary Intervention (PCI) admission can predict the risk of readmission.

\begin{abstract}
Background-Reducing readmissions following hospitalization is a national priority. Identifying patients at high risk for readmission after PCI early in a hospitalization would enable hospitals to enhance discharge planning.
\end{abstract}

Methods-We developed 3 different models to predict 30-day inpatient readmission to our institution for patients who underwent PCI between January 2010 and April 2013. These models used data available: 1) at admission, 2) at discharge 3) from CathPCI Registry data. We used logistic regression and assessed the discrimination of each model using the c-index. The models were validated with testing on a different patient cohort who underwent PCI between May 2013 and September 2015.

\begin{abstract}
Results-Our cohort included 6,717 PCI patients; 3,739 in the derivation cohort and 2,978 in the validation cohort. The discriminative ability of the admission model was good (C-index of 0.727). The c-indices for the discharge and cath PCI models were slightly better. (C-index of 0.751 and 0.752 respectively). Internal validation of the models showed a reasonable discriminative admission model with slight improvement with adding discharge and registry data (C-index of $0.720,0.739$ and 0.741 respectively). Similarly validation of the models on the validation cohort showed similar results (C-index of $0.703,0.725$ and 0.719 respectively).
\end{abstract}

Conclusion-Simple models based on available demographic and clinical data may be sufficient to identify patients at highest risk of readmission following PCI early in their hospitalization.

Corresponding Author: Zaher Fanari, M.D., Prairie Heart Institute, Springfield, IL, 619 E Mason St, Springfield, IL 62769. Tel: +1 (314) 808-1610 Fax: +1 (913) 588-6010, zfanari@gmail.com.

Disclosure:

None of the authors has any conflict of interest to disclose. 


\section{Keywords}

Readmission; Risk Prediction; Percutaneous Coronary Intervention

\section{Introduction}

Percutaneous coronary intervention (PCI) is among the most common major medical procedures in the U.S. ${ }^{1}$ Despite the improvement of care over time, a significant number of Medicare patients (14.6\%) are readmitted after PCI. ${ }^{2,3}$ The Patient Protection and Affordable Care Act linked many quality outcomes including 30-day readmission rates to hospital reimbursement. ${ }^{4}$ The Center for Medicare and Medicaid Services (CMS) publicly reports hospital level 30-day readmission rates for heart failure (HF), acute myocardial infarction (AMI. ${ }^{1,5,6}$ As a quality measure many hospitals are interested not only in reducing readmissions after AMI or HF exacerbation, but also after elective procedure like PCI.

With these changing incentives, many health systems are developing programs to improve the quality of transitional and longitudinal care upon discharge. Research suggests that multi-component interventions focused on transitional care can reduce the readmission rates in certain conditions, including PCI ${ }^{7}$. However, for these programs to be cost-effective, it will be necessary to target efforts to the patients that are most likely to benefit from the increased intensity of services. Additionally, services may be most effective if they begin during the hospitalization so that the care quality and patient education can be maximized during the hospital stay. ${ }^{5}$

Although many studies have identified strong predictors for readmissions risk following PCI, ${ }^{3,8,9}$ to date there are only two risk model was designed so far to specifically predict readmission risk for patients undergoing PCI. ${ }^{10,11}$ However although these risk models used registry data available early in the admission or discharge to identify patients at high risk for readmission, they did not address the possibility of using data readily available prior to or at the time of admission to predict readmission risk at the minute of index admission.

We sought to develop a model to predict readmission risk following PCI using clinical and administrative data available within our hospital system at the time of admission, and to determine the incremental benefit to risk assessment of adding 1) clinical information available at the time of discharge, and 2) registry data from the CathPCI Registry. Our findings will inform the use of clinical data within hospital systems to prospectively riskstratify patients to support the cost-effective application of care management or other resources with the intent to reduce readmission.

\section{Methods}

\section{Study Design, Population, and Setting}

We conducted a retrospective cohort of all patients with revascularization with PCI at Christiana Care Health System between January 1, 2010 and September 30, 2015. Christiana Care is a large system that comprises two hospitals with more than 1,100 beds as well as a 
variety of outpatient and other services in facilities and provides the majority of cardiovascular care in Delaware and the surrounding area with an estimate of $1700 \mathrm{PCI}$ and more than 600 open heart surgery procedures annually. We identified all patients who were discharged alive following PCI. We further divided the cohort into 1) a derivation cohort that was used to develop the 3 separate prediction models and included PCI patients admitted between April 1, 2010 and April 30,2013; and 2) a validation cohort that was used to test the prediction models and included those admitted between May 1, 2013 and September 30, 2015. Importantly, the validation cohort included patients that were included in a longitudinal care management program for patients following coronary revascularization. Patients were enrolled in the program during the hospitalization and followed with telephonic care management following discharge. The Christiana Care Institutional Review Board approved the study.

\section{Outcomes}

We identified inpatient, non-elective readmissions to Christiana Care within 30 days of discharge from the index procedure. We excluded elective readmissions such as staged PCIs based on admission records. We excluded patients who were who received coronary artery bypass surgery during the same admission. We identified readmissions at our own system and we used QualityNet Data from CMS to identify readmissions at other hospitals.

\section{Candidate Variables and Model Derivation}

Candidate variables for the prediction model were drawn from three sources: 1) administrative and billing data from the data warehouse at Christiana Care (demographics, previous utilization, and comorbidities; 2) clinical data including initial and discharge laboratory and vital signs from key clinical systems; and 3) registry information from the NCDR CathPCI Registry for data concerning anatomic and procedural information. Comorbidities were classified from administrative data using the Elixhauser classification. ${ }^{12}$

In order to determine the incremental value of additional information gathered across the hospital visit, we developed the following three models that sequentially added information available during the hospitalization: 1) an admission model that included only variables available at the time the patient arrived at the hospital, 2) a discharge model that included administrative and clinical information available at the time of discharge; and 3) a discharge model that also included anatomic and procedural information from the CathPCI Registry. This progression of models was chosen based on the timing of availability of this data in the clinical setting. The CathPCI registry information, for example, is collected by staff following discharge and is not available for operational purposes at the time of discharge. These variables and the progress in building each model are shown in Table 1.

Hierarchical logistic regression was used to model readmissions (a patient may have had more than one PCI during the study period). Derivation models were developed by a combination of forward selection and backward elimination of variables. Variables were entered if $\mathrm{p} \leq .2$ and removed if $\mathrm{p}>.2$. Reduced models were compared to larger models by likelihood ratio tests. Fractional polynomial (FP) regression was used to assess non-linearity of continuous variables. Cubic splines were then used to determine categories for nonlinear 
continuous variables. Although adding variables to the sequential models will likely change the estimation of odds ratios (as well as contribution to the predictive ability of the model), variables were retained in subsequent models regardless of their contribution to predictive ability. Model discrimination was assessed by the c-statistic and model calibration was assessed by plotting observed readmission rates with deciles of model-predicted rates.

Models were developed for PCI patients admitted to the hospital between April 1, 2010 and April 30, 2013. Internal model validity was assessed by bootstrap methods -500 bootstrap replicates with replacement were drawn to calculate bias-corrected c-indices. The derivation models were then applied to patients admitted between May 1, 2013 and September 30, 2015 to assess external validity.

\section{Results}

The total number of PCI patients was 6,717 including 3,739 in the derivation cohort and 2,978 in the validation cohort. These patients had a total of 7,749 hospitalizations; 4,340 in the derivation cohort and 3,409 in the validation cohort. The readmission rate was $8.4 \%$. The 30-days readmission rate was $8.5 \%$ and The 30 -days mortality was very low at $0.5 \%$. Table-2 shows the demographic and clinical characteristics of the derivation and validation cohorts.

The admission, admission + discharge and admission + discharge + cath PCI registry models are presented in Tables 3-5 respectively. Increasing age, female gender and previous hospitalization within 6 months of the current hospitalization were risk factors for readmission. Whereas private insurance carrier and elective procedure were predictive for lower risk of readmission. The c-index for the admission model was 0.727 (95\% CI $=0.697$ - 0.757). The addition of data available at discharge, including total count of Elixhauser comorbidities as well as the presence of $\mathrm{CHF}$, renal failure and depression, and discharge to skilled nursing facility (SNF) resulted in a statistically significant increase $(\mathrm{p}<0.001)$ in cindex to $0.751(95 \% \mathrm{CI}=0.734-0.780)$. The only variable from the cath PCI registry to be added to the admission + discharge model was Canadian Classification System angina class. However, the impact on the model performance was negligible, with a c-index of 0.752 $(95 \% \mathrm{CI}=0.724-0.781)$ and did not statistically differ from the admission + discharge model $(\mathrm{p}=0.805)$.

Internal validation of the admission, admission + discharge and admission + discharge + registry models resulted in c-indices of $0.720,0.739$ and 0.741 respectively. External validation of the admission, admission + discharge and admission + discharge + registry models resulted in c-indices of $0.703,0.725$ and 0.719 respectively. Figures $1 \mathrm{~A}-\mathrm{C}$ shows calibration plots for each of the models for the derivation and the validation cohorts.

\section{Discussion}

We developed 3 models including an early model at admission and two late models at discharge. The early risk prediction model was reasonably predictive for readmission risk, and adding data available at discharge or additional data from CathPCI registry only minimally improved the performance of the initial model. The addition of details regarding 
anatomical (i.e. the number or location of lesions involved) or procedural variants (i.e. access site or complications) did not add to the model performance. Our findings suggest that a simple model comprised of data available at hospital admission may offer a reasonable predication of readmission risk close to more robust models using discharge data. A simple early model may be used to help physicians and hospitals in designing early interventions and better utilization of resources that may help in reducing the readmission rate. Hospitals will be able to direct case management personnel to better coordinate more robust counseling about discharge planning, new medications, potential expectations for post discharge care at home, arranging for home health or referral rehabilitation services at home or at rehabilitation facilities, and setting a closer and more frequent outpatient follow-up. Case management teams have been shown to an effective strategy for reducing readmissions and associated costs especially if it was directed towards those who are at higher risk for readmission. ${ }^{13}$

There is an increased need for reducing readmission rates nationwide, especially with pressure from the Affordable Care Act with its financial incentives for hospitals to minimize readmissions and the fact that percutaneous coronary intervention (PCI) became a target in $2015 .{ }^{10}$ Several studies have identified patients who are at higher risk for readmissions after PCI. ${ }^{3,8,9}$ Administrative data derived from Medicare claims of 315,241 patients showed increased risk for readmission after PCI to be associated with female sex, older age, diabetes mellitus, heart failure, renal failure, previous ischemic heart disease, and acute MI. ${ }^{3}$ The New York State registry data of 40,093 PCI patients showed the risk of readmission to be linked to age, depressed ejection fraction, multivessel disease, recent MI, peripheral vascular disease, chronic obstructive pulmonary disease, diabetes mellitus, and worsening renal failure or dialysis. ${ }^{8}$ Whereas data from a prospective registry from Saint Marys Hospital in Minnesota showed the risk of 30 days readmission to be related to female sex, Medicare insurance, less than high school education, unstable angina, moderate-to-severe renal disease, cerebrovascular incident or transient ischemic attack, moderate-to-severe renal disease, chronic obstructive pulmonary disease, peptic ulcer disease, metastatic cancer, and a length of stay $>3$ days. ${ }^{9}$ Readmission rates vary in these studies from $9.6 \%$ to $15.6 \%$ with wide variations among hospitals in the US as reported by Medicare data between $8.9 \%-$ $22 \% .3,8,9$

Risk predictions models were developed to predict readmission rate in the general hospital population with LACE index score and HOSPITAL Score. Both models had fair discriminative ability with C- statistics of 0.68 and 0.71 respectively, using data available only at discharge, therefore limiting the opportunity for early interventions to reduce readmission risk. ${ }^{14,15}$ Multiple models have been developed to predict readmission in heart failure patients or after acute myocardial infarction. ${ }^{16-18}$ But such models risk stratify a higher risk population, use many candidate variables that are available only at discharge, and may not be applicable in predicting risk for readmission after PCI. ${ }^{18}$ Furthermore; the Cindex of these model was $0.60-0.63 .{ }^{19}$

The first and so far only specific risk prediction model for readmissions after PCI based on admission data was derived by Wasfy et al $^{10}$ for the Massachusetts Department of Public Health using the National Cardiovascular Data Registry (NCDR) Cath-PCI data collection 
instrument. Two separate models were developed, with one designed to predict risk of readmission early before PCI and the other to predict risk of readmission at discharge. Similarly to our models, adding data at discharge did not significantly impact the predictive discrimination ability with $\mathrm{C}$-index increase from 0.68 to 0.69 . Another prediction model was build based on Medicare patients' registry data to predict readmission risk. ${ }^{11}$ These prediction models did show that early data at admission might be predictive of readmission risk. However, these models had some limitations. First, they did not add previous utilization as an important potential predictor of readmission. Second, they used registry data that require abstracting and that may be subjective to inaccurate coding and/or not inclusive of all co-morbidities and complications that may impact and predict readmission. Third, the early model included some variables that may not be completely available at the time of the admission and not available before PCI when urgent revascularization is required, Finally, even the early admission data are derived from registry sources that usually are collected either late in the admission or even after discharge, and therefore may not offer the opportunity to identify those at risk during the course of the index admission. There is increased evidence that patient level data from hospital medical records can be more predictive than those derived from administrative or registry data and can help in improving the predictively of risk prediction models. ${ }^{20}$

In comparison to the previously available models, our risk models had multiple advantages. First, these models provide the chance to predict the risk of readmissions at the moment of admission even before any available vitals or labs, and this early recognition helps in planning the admission and potential interventions to those at risk as early as possible to prevent readmissions. Second, they showed that general risk factors including demographic data and previous utilization may be the strongest determinant of readmission risk, Third, they showed that patient specific data are way more important in predicting the risk of readmission than procedure related factors. It is an important finding that anatomical or procedural factors did not really add to the predictability of readmission risk. Fourth, they used readily available hospital/outpatient-level clinical and demographic data that can be easily obtained electronically by preset tools without the need for chart review and abstracting. Fifth, they showed that adding registry data with complicated information did not add much to more general measures of comorbidity and risk. Finally, they showed higher discrimination ability than previously available models and therefore it would be more predictive of the risk of readmission.

There are multiple limitations to our work. First, data are derived from a single center and our readmission rates only include visits back to our hospital system. However, our system provides the overwhelming majority of care for our region and a care management implemented at the time of the study provided data on readmission in other hospitals. Our findings and observed patterns of care may not be completely generalizable to other hospitals settings, particularly those with lower degrees of market capture. Second, although the models were validated internally, our validation population was subject to a care management intervention that may impact the utilization in that population. External validation of our model or models developed using similar approaches would be helpful to prove applicability to different PCI patient populations. Finally, although we tried to make the models parsimonious, as readmission risk is multifactorial and not dependent on a small 
number of strong predictors, the models still include a large number of variables $(9,14$, and 16) to retain sufficient predictive ability. However, we believe that both web based and other information technologies tools can ease their implementation in routine clinical care. Also our admission model uses readily available data at admission to predict those at risk when it is pertinent and useful.

\title{
Conclusions
}

We demonstrated that a model based on clinical data available at the time of admission has sufficient discrimination and calibration to predict those at risk for readmission after PCI and is only marginally improved by adding more complete clinical data. Adding administrative, and registry data on top of already available clinical data did not increase predictively of readmission risk.

Models such as this one could allow providers and health systems to target high-risk patients with enhanced discharge planning during the course of the hospitalization, and this may improve the ability to avoid readmissions in a cost-effective manner. Future work should explore the validation of this approach in other settings.

\section{Acknowledgments}

\author{
Funding: \\ Supported by grant number 1C1CMS331027 from the Department of Health and Human Services, Centers for \\ Medicare \& Medicaid Services (PI: Weintraub) and by an Institutional Development Award (IDeA) from the \\ National Institute of General Medical Sciences of the National Institutes of Health grant number U54-GM104941 \\ (PI: Binder-Macleod). The contents of this publication are solely the responsibility of the authors and do not \\ necessarily represent the official views of the U.S. Department of Health and Human Services or any of its \\ agencies. The research presented here was conducted by the awardee. Findings might or might not be consistent \\ with or confirmed by the findings of the independent evaluation contractor.
}

\section{References}

1. Kereiakes DJ. Return to sender hospital readmission after percutaneous coronary intervention. J Am Coll Cardiol. 2009; 54:908-910. [PubMed: 19712800]

2. U.S. Department of health and human services. [Accessed june 9, 2014] Administration implements new health reform provision to improve care quality, lower costs. Http://www.Healthcare.Gov/news/ factsheets/2011/04/valuebasedpurchasing04292011a.Html

3. Curtis JP, Schreiner G, Wang Y, Chen J, Spertus JA, Rumsfeld JS, et al. All-cause readmission and repeat revascularization after percutaneous coronary intervention in a cohort of medicare patients. $\mathrm{J}$ Am Coll Cardiol. 2009; 54:903-907. [PubMed: 19712799]

4. Patient protection and affordable care act of 2010. Publ no. 111-148. 124 stat 119.

5. Krumholz HM, Normand SL, Spertus JA, Shahian DM, Bradley EH. Measuring performance for treating heart attacks and heart failure: The case for outcomes measurement. Health Aff. 2007; 26:75-85.

6. Krumholz HM, Normand SL. Public reporting of 30-day mortality for patients hospitalized with acute myocardial infarction and heart failure. Circulation. 2008; 118:1394-1397. [PubMed: 18725492]

7. Naylor MD, Aiken LH, Kurtzman ET, Olds DM, Hirschman KB. The care span: The importance of transitional care in achieving health reform. Health Aff. 2011; 30:746-754. 
8. Hannan EL, Zhong Y, Krumholz H, Walford G, Holmes DR Jr, Stamato NJ, et al. 30-day readmission for patients undergoing percutaneous coronary interventions in new york state. $\mathrm{J}$ Am Coll Cardiol Interv. 2011; 4:1335-1342.

9. Khawaja FJ, Shah ND, Lennon RJ, Slusser JP, Alkatib AA, Rihal CS, et al. Factors associated with 30-day readmission rates after percutaneous coronary intervention. Arch Intern Med. 2012; 172:112-117. [PubMed: 22123752]

10. Wasfy JH, Rosenfield K, Zelevinsky K, Sakhuja R, Lovett A, Spertus JA, et al. A prediction model to identify patients at high risk for 30-day readmission after percutaneous coronary intervention. Circ Cardiovasc Qual Outcomes. 2013; 6:429-435. [PubMed: 23819957]

11. Minges KHJ, Fiorilli PN, Curtis JP. Development and validation of a simple risk score to predict 30-day readmission after percutaneous coronary intervention in a cohort of medicare patients. Catheter Cardiovasc Interv. 2016

12. Southern DA, Quan H, Ghali WA. Comparison of the elixhauser and charlson/deyo methods of comorbidity measurement in administrative data. Med Care. 2004; 42:355-360. [PubMed: 15076812]

13. Kolbasovsky A, Zeitlin J, Gillespie W. Impact of point-of-care case management on readmissions and costs. Am J Manag Care. 2012; 18:e300-306. [PubMed: 22928799]

14. van Walraven C, Dhalla IA, Bell C, Etchells E, Stiell IG, Zarnke K, et al. Derivation and validation of an index to predict early death or unplanned readmission after discharge from hospital to the community. CMAJ. 2010; 182:551-557. [PubMed: 20194559]

15. Donze J, Aujesky D, Williams D, Schnipper JL. Potentially avoidable 30-day hospital readmissions in medical patients: Derivation and validation of a prediction model. JAMA Intern Med. 2013; 173:632-638. [PubMed: 23529115]

16. Philbin EF, DiSalvo TG. Prediction of hospital readmission for heart failure: Development of a simple risk score based on administrative data. J Am Coll Cardiol. 1999; 33:1560-1566. [PubMed: 10334424]

17. Yamokoski LM, Hasselblad V, Moser DK, Binanay C, Conway GA, Glotzer JM, et al. Prediction of rehospitalization and death in severe heart failure by physicians and nurses of the escape trial. $\mathrm{J}$ Card Fail. 2007; 13:8-13. [PubMed: 17338997]

18. Krumholz HM, Lin Z, Drye EE, Desai MM, Han LF, Rapp MT, et al. An administrative claims measure suitable for profiling hospital performance based on 30-day all-cause readmission rates among patients with acute myocardial infarction. Circ Cardiovasc Qual Outcomes. 2011; 4:243252. [PubMed: 21406673]

19. Lloyd-Jones DM. Cardiovascular risk prediction: Basic concepts, current status, and future directions. Circulation. 2010; 121:1768-1777. [PubMed: 20404268]

20. Wasfy JH, Singal G, O'Brien C, Blumenthal DM, Kennedy KF, Strom JB, et al. Enhancing the prediction of 30-day readmission after percutaneous coronary intervention using data extracted by querying of the electronic health record. Circ Cardiovasc Qual Outcomes. 2015; 8:477-485.

[PubMed: 26286871] 

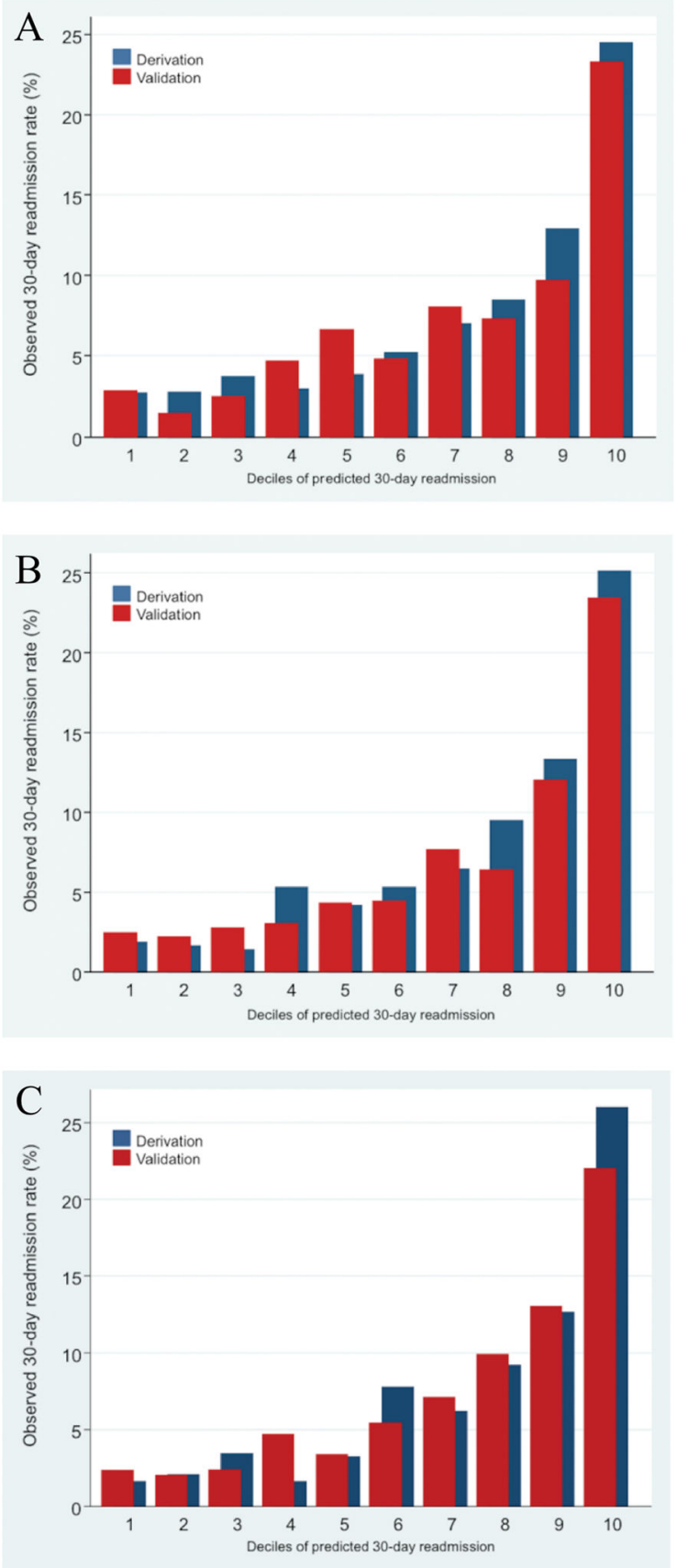

Figure 1.

A- Calibration Plot Of Admission Model For PCI And Application To Validation Data. B- Calibration Plots For Admission + Discharge Model For PCI And Application To Validation Data.

C- Calibration Plots For Admission + Discharge + Registry Model For PCI And Application To Validation Data. 


\section{Table 1}

Variables by Data class and Sequential model development.

\begin{tabular}{|c|c|c|}
\hline \multicolumn{3}{|l|}{ Variables By Data Class } \\
\hline Baseline / Admission & Discharge & Cath PCI Registry \\
\hline Age & Length Of Stay & Any Complication \\
\hline Sex & AMI Indication & LVEF At Discharge \\
\hline Race & Any ICU Stay & Pre-TIMI Flow \\
\hline Insurance & Weekend Discharge & Cerebral Vascular Disease \\
\hline \multirow[t]{5}{*}{ Elective Status } & \multirow{5}{*}{$\begin{array}{l}\text { Discharge Location } \\
\text { Home } \\
\text { Home With Services } \\
\text { Skilled Nursing Facility } \\
\text { Others Facility }\end{array}$} & Artery Access Location: \\
\hline & & Femoral \\
\hline & & Radial \\
\hline & & Other \\
\hline & & Lesion Count $\geq 2$ \\
\hline Previous PCI & \multirow{6}{*}{$\begin{array}{cl}\text { Elixhauser Comorbidities } \\
\text { • } & \text { Comorbidity Count } \\
\text { - } & \text { CHF } \\
\text { - } & \text { COPD } \\
\text { - } & \text { Diabetes } \\
\text { - } & \text { Renal Failure } \\
\text { - } & \text { Perivascular Disease } \\
\text { - } & \text { Valve Disease } \\
\text { - } & \text { Electrolyte Imbalance } \\
\text { - } & \text { Obesity }\end{array}$} & High Lesion Complexity \\
\hline Previous CABG & & Beta Blocker \\
\hline Weekend Admission & & ACEI/ARB \\
\hline Previous Hospitalization & & Antiplatelet Type (Clopidogrel/Prasugrel/ Ticagrelor) \\
\hline \multirow[t]{2}{*}{ Previous AMI } & & Canadian Classification System Angina Class \\
\hline & & $\begin{array}{l}\text { Angina Type: } \\
\text { No Symptoms } \\
\text { Stable Angina } \\
\text { Unstable Angina } \\
\text { NSTEMI } \\
\text { STEMI }\end{array}$ \\
\hline Model & \multicolumn{2}{|l|}{ Data Classes Included } \\
\hline 1 & \multicolumn{2}{|l|}{ Baseline - Admission } \\
\hline 2 & \multicolumn{2}{|l|}{ Baseline - Admission / Discharge } \\
\hline 3 & \multicolumn{2}{|c|}{ Baseline - Admission / Discharge / Cath PCI Registry } \\
\hline
\end{tabular}

All variables in each set were initially entered into the model and then removed by elimination criteria.

Variables retained in each model were retained in the subsequent model.

Abbreviations: PCI= Percutaneous Intervention; AMI= Acute Myocardial Infarction; LVEF= Left Ventricle Ejection Fraction; ICU= Intensive Care Unit; TIMI= Thrombolysis in Myocardial Infarction; ACEI= Angiotensin Converting Enzyme Inhibitor; ARB= Angiotensin Receptor Inhibitor; $\mathrm{CABG}=$ Coronary Artery Bypass Grafting; $\mathrm{COPD}=$ Chronic Obstructive Pulmonary Disease. NSTEMI= Non-ST Elevation Myocardial Infarction; STEMI= ST Elevation Myocardial Infarction. 
Table 2

PCI Derivation and Validation Cohorts Demographic and Clinical Characteristics.

\begin{tabular}{|c|c|c|c|}
\hline \multicolumn{2}{|l|}{ Variable } & $\begin{array}{l}\text { Derivation cohort } \\
\quad \mathbf{N}=\mathbf{3 , 7 3 9}\end{array}$ & $\begin{array}{l}\text { Validation cohor } \\
\quad \mathbf{N}=\mathbf{2 , 9 7 8}\end{array}$ \\
\hline \multicolumn{2}{|l|}{ Age (years) } & $64.2 \pm 12.1$ & $64.4 \pm 11.9$ \\
\hline \multicolumn{2}{|l|}{ Female (\%) } & 31.3 & 31.8 \\
\hline \multirow{3}{*}{ Race $(\%)$} & White & 82.4 & 82.0 \\
\hline & Black & 12.8 & 12.9 \\
\hline & Other & 4.8 & 5.1 \\
\hline \multirow[t]{5}{*}{ Insurance (\%) } & Medicare & 51.2 & 52.5 \\
\hline & Medicaid & 5.9 & 7.9 \\
\hline & Private & 39.0 & 35.8 \\
\hline & Self pay & 2.8 & 0.8 \\
\hline & Other & 1.1 & 2.9 \\
\hline \multicolumn{2}{|l|}{ Elective (\%) } & 37.0 & 35.5 \\
\hline \multicolumn{2}{|l|}{ Transfer $(\%)$} & 7.5 & 5.0 \\
\hline \multicolumn{2}{|l|}{ Previous AMI } & 11.4 & 11.4 \\
\hline \multicolumn{2}{|l|}{ Previous PCI } & 36.2 & 36.6 \\
\hline \multicolumn{2}{|l|}{ Previous CABG } & 9.6 & 8.9 \\
\hline \multirow[t]{3}{*}{ Previous IOE hospitalization within 6 mos. (\%) } & None & 70.9 & 69.0 \\
\hline & 1 & 19.4 & 19.4 \\
\hline & $>1$ & 9.7 & 11.6 \\
\hline \multirow[t]{5}{*}{ Comorbidities } & Total count & $3.4 \pm 2.8$ & $3.7 \pm 2.9$ \\
\hline & $\mathrm{CHF}(\%)$ & 16.6 & 18.9 \\
\hline & $\operatorname{COPD}(\%)$ & 21.4 & 22.6 \\
\hline & Diabetes (\%) & 38.7 & 41.9 \\
\hline & Renal failure $(\%)$ & 14.1 & 14.0 \\
\hline \multicolumn{2}{|l|}{ LOS (days) } & $2.8 \pm 3.6$ & $2.7 \pm 4.4$ \\
\hline \multirow[t]{4}{*}{ Discharge disposition (\%) } & Home & 90.1 & 86.7 \\
\hline & Home w/ services & 6.3 & 9.4 \\
\hline & SNF & 2.3 & 2.0 \\
\hline & Other facility & 1.4 & 1.9 \\
\hline
\end{tabular}

Gender and race percentages are based on number of patients; all other categorical variables on number of hospitalizations.

Age, comorbidity count and LOS are mean \pm 1 standard deviation 
Table 3

PCI Admission Model Predicting 30-Day Non-Elective Inpatient Readmission.

\begin{tabular}{|l|l|l|l|l|}
\hline \multicolumn{2}{|l|}{ Variable } & Odds Ratio & 95\% Confidence Interval & P Value \\
\hline \multicolumn{2}{|l|}{ Age (10-Year Increments) } & 1.26 & $1.11-1.42$ & $<.001$ \\
\hline \multicolumn{2}{|l|}{ Female } & 1.27 & $0.99-1.62$ & .06 \\
\hline \multirow{3}{*}{ Race } & White & 1.00 & ------------- & --------- \\
\cline { 2 - 5 } & Black & 0.64 & $0.43-0.94$ & .025 \\
\cline { 2 - 5 } & Other & 0.65 & $0.33-1.26$ & .20 \\
\hline Private Insurance & 0.75 & $0.54-1.06$ & .10 \\
\hline \multicolumn{2}{|l|}{ Elective } & 0.35 & $0.26-0.47$ & $<.001$ \\
\hline \multicolumn{2}{|l|}{ Previous Hospitalization Within 6 Months } & 1.46 & $1.32-1.62$ & $<.001$ \\
\hline
\end{tabular}




\section{Table 4}

PCI Admission + Discharge Model Predicting 30-Day Non-Elective Inpatient Readmission.

\begin{tabular}{|c|c|c|c|c|}
\hline \multicolumn{2}{|l|}{ Variable } & Odds ratio & $95 \%$ confidence interval & p value \\
\hline \multicolumn{2}{|l|}{ Age (10-Year Increments) } & 1.20 & $1.05-1.37$ & .002 \\
\hline \multicolumn{2}{|l|}{ Female } & 1.10 & $0.85-1.45$ & .47 \\
\hline \multirow[t]{3}{*}{ Race } & White & 1.00 & -------------- & --------- \\
\hline & Black & 0.59 & $0.40-0.88$ & .01 \\
\hline & Other & 0.70 & $0.37-1.35$ & .23 \\
\hline \multicolumn{2}{|l|}{ Private Insurance } & 0.94 & $0.67-1.33$ & .74 \\
\hline \multicolumn{2}{|l|}{ Elective } & 0.41 & $0.30-0.56$ & $<.001$ \\
\hline \multicolumn{2}{|c|}{ Previous Hospitalization Within 6 Months } & 1.26 & $1.13-1.39$ & $<.001$ \\
\hline \multirow[t]{4}{*}{ Elixhauser Co-Morbidities } & Total Count & 1.07 & $1.01-1.14$ & .03 \\
\hline & Congestive Heart Failure & 1.44 & $1.01-2.04$ & .04 \\
\hline & Renal Failure & 1.34 & $0.91-1.98$ & .14 \\
\hline & Depression & 1.26 & $0.90-1.77$ & .17 \\
\hline \multicolumn{2}{|c|}{ Discharge To Skilled Nursing Facility } & 1.53 & $1.06-2.23$ & .02 \\
\hline
\end{tabular}




\section{Table 5}

PCI Admission + Discharge + Registry Model Predicting 30-Day Non-Elective Inpatient Readmission.

\begin{tabular}{|c|c|c|c|c|}
\hline \multicolumn{2}{|l|}{ Variable } & Odds Ratio & 95\% Confidence Interval & P Value \\
\hline \multicolumn{2}{|l|}{ Age (10-Year Increments) } & 1.24 & $1.08-1.41$ & .002 \\
\hline \multicolumn{2}{|l|}{ Female } & 1.16 & $0.89-1.51$ & .28 \\
\hline \multirow[t]{3}{*}{ Race } & White & 1.00 & ----------------- & --------- \\
\hline & Black & 0.59 & $0.39-0.87$ & .01 \\
\hline & Other & 0.71 & $0.37-1.37$ & .31 \\
\hline \multicolumn{2}{|l|}{ Private Insurance } & 0.94 & $0.67-1.32$ & .73 \\
\hline \multicolumn{2}{|l|}{ Elective } & 0.37 & $0.27-0.50$ & $<.001$ \\
\hline \multicolumn{2}{|l|}{ Previous Hospitalization Within 6 Months } & 1.26 & $1.14-1.39$ & $<.001$ \\
\hline \multirow[t]{4}{*}{ Elixhauser Co-morbidities } & Total Count & 1.08 & $1.01-1.15$ & .02 \\
\hline & Congestive Heart Failure & 1.54 & $1.10-2.17$ & 0.01 \\
\hline & Renal Failure & 1.37 & $0.93-2.01$ & 0.12 \\
\hline & Depression & 1.22 & $0.87-1.72$ & 0.24 \\
\hline \multicolumn{2}{|l|}{ Discharge To Skilled Nursing Facility } & 0.61 & $0.33-1.15$ & 0.13 \\
\hline \multirow[t]{2}{*}{ Canadian Classification System Angina Class } & Class II & 1.42 & $1.05-1.93$ & 0.02 \\
\hline & Class III & 1.31 & $0.98-1.75$ & 0.07 \\
\hline
\end{tabular}

